\title{
ORIENTATION DEPENDENT SPIN-LATTICE RELAXATION IN CdMnSe*
}

\author{
A.M. Witowski \\ Institute of Experimental Physics, Warsaw University \\ Hoża 69, 00-681 Warszawa, Poland \\ T. STRUTZ ${ }^{\dagger}$ AND P. Wyder \\ High Magnetic Field Laboratory Grenoble, MPI FKF \& CNRS \\ 38042 Grenoble Cedex 9, France
}

The magnetization dynamics of $\mathrm{Cd}_{1-x} \mathrm{Mn}_{x}$ Se at $4.5 \mathrm{~K}$ as a function of magnetic field up to $22 \mathrm{~T}$ was measured using a nonresonant technique. For $x=0.01$ the relaxation does not depend on orientation and in higher fields the relaxation rates are proportional to $B^{3}$. For $x=0.02$ a dependence on orientation is observed. The difference does not depend on magnetic field. This suggests that either the interaction between Mn ions responsible for spin-lattice relaxation in Mn clusters is anisotropic, or the relaxing clusters are oriented in a given manner with respect to the $c$ axis.

PACS numbers: 76.30.Fc, 63.20.Dj

Using the nonresonant technique described earlier [1], we measured the magnetization dynamics in $\mathrm{Cd}_{1-x} \mathrm{Mn}_{x}$ Se at $4.5 \mathrm{~K}$ as a function of magnetic field. Two different compositions were investigated, namely $x=0.01$ and $x=0.02$. The measurements were done for external magnetic field $B$ both: parallel and perpendicular to the $c$ axis (the CdSe host crystal, as well as mixed CdMnSe crystals, has a wurtzite structure). YAG:Nd laser pulses $(\lambda=1.06 \mu \mathrm{m})$ of $10 \mathrm{~ns}$ duration were used to create the thermal inequilibrium between spins and the lattice [1], rising lattice temperature. The spins then warm up to reach thermal equilibrium with the "warm" lattice. It causes the magnetization changes which are detected with a pick-up coil.

The measured spin-lattice relaxation (SLR) rates are dependent on the external magnetic field, temperature, and composition. For lower Mn composition the data do not show any orientation dependence. In low magnetic field the relaxation rates (RR) weakly increase with $B$. In higher fields their dependence is not in contradiction with expected $B^{3}$ dependence (see Fig. 1). Such a magnetic field de-

*This work is partially supported by the State Committee for Scientific Research (Republic of Poland) throughout grant \#2 24109203.

tCurrent address: RCAST, University of Tokyo, Tokyo 153, Japan. 


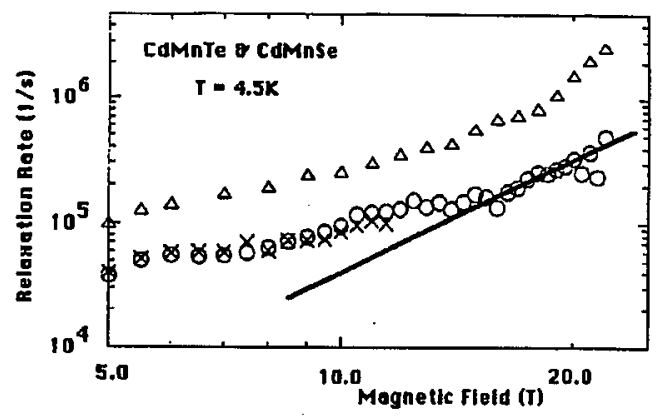

Fig. 1. The relaxation rate for $\mathrm{Cd}_{0.99} \mathrm{Mn}_{0.01} \mathrm{Se}$ for external field parallel (circles) and perpendicular (crosses) to the $c$ axis and $\mathrm{Cd}_{0.998} \mathrm{Mn}_{0.002} \mathrm{Te}$ (triangles) as a function of magnetic field measured at $4.5 \mathrm{~K}$. The solid line represents a $B^{3}$ dependence.

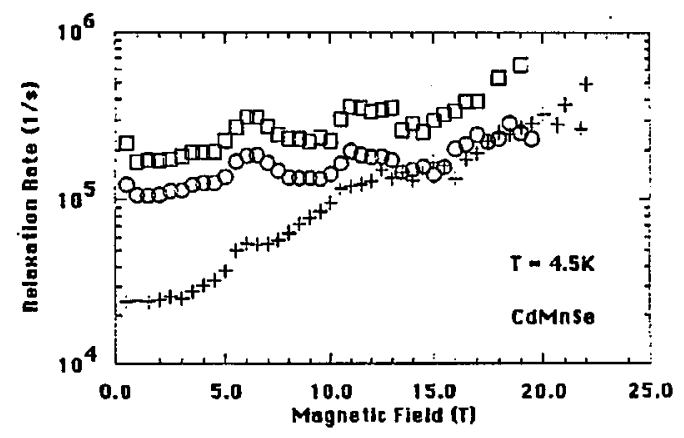

Fig. 2. The relaxation rate for $\mathrm{Cd}_{0.98} \mathrm{Mn}_{0.02}$ Se for external field parallel (circles) and perpendicular (squares) to the $c$ axis vs. magnetic field measured at $4.5 \mathrm{~K}$. For comparison, data for $\mathrm{Cd}_{0.99} \mathrm{Mn}_{0.01} \mathrm{Se}$ with the $c$ axis parallel to the external field are also plotted (crosses).

pendence $\left(B^{3}\right)$ is typical of relaxation with emission and absorption of one phonon (so-called direct process), whose density of states can be described by the Debye approximation (see e.g. [2]). The observed rates are smaller than in $\mathrm{Cd}_{1-x} \mathrm{Mn}_{x} \mathrm{Te}$ with the same compositions [3] (the relaxation rates for $\mathrm{Cd}_{0.99} \mathrm{Mn}_{0.01} \mathrm{Te}$ have almost the same value as rates for $\left.\mathrm{Cd}_{0.998} \mathrm{Mn}_{0.002} \mathrm{Te}\right)$. Also in high magnetic fields the magnetic field dependence is stronger for $\mathrm{Cd}_{1-x} \mathrm{Mn}_{x}$ Te (Fig. 1). This is easily explained since far-infrared data on Mn local acoustic vibrations show that the peak of density of TA phonon states is at energy about $50 \%$ higher in CdSe than in $\mathrm{CdTe}[4,5]$. Therefore, in the investigated magnetic field range the density of phonon states (DoPS) can be described by the Debye approximation for CdSe but not for CdTe [6]. This can probably also partially explain the differences of the values of relaxation rates. The DoPS for a given energy at a higher magnetic field is higher for CdTe, because it is closer to the peak in the DoPS for TA phonons.

For $\mathrm{Cd}_{1-x} \mathrm{Mn}_{x}$ Se with $x=0.02$, as shown in Fig. 2, the relaxation rates are larger than for $x=0.01$, which suggests that for $x=0-22$ clusters of interacting 
magnetic ions play a more important role in the magnetization relaxation process than for the lower composition. A dependence of relaxation rates on orientation is also observed (Fig. 2). The difference in absolute values increases with increasing magnetic field (note semilogarithmic scale). For both orientations at fields where the exchange Mn pair levels cross (about $13 \mathrm{~T}$ ) double structures are observed in agreement with previously reported data [7]. Where the levels cross, mixing of the states occurs due to the anisotropic Dzialoshynski-Moriya type exchange interaction. This allows for an effective spin-lattice relaxation due to the modulation of the above-mentioned exchange interaction. Because of the existence of two types of pairs in the wurtzite type crystallographic structure, a double structure in the relaxation is observed [7]. We have no explanation for the structure at $7 \mathrm{~T}$.

Because the anisotropy of the relaxation rate is observed for higher Mn compositions only, one can assume that the magnetization relaxation is governed by the relaxation in Mn clusters, which also interact via an anisotropic interaction (e.g. a Dzialoshynski-Moriya type exchange). In such a case, the mechanism of spin-lattice relaxation is phonon modulation of the interaction. The possibility that dominant clusters have a given orientation with respect to the $c$ axis should also be taken into consideration.

\section{Acknowledgment}

One of us (A.M.W.) acknowledges the financial support from the European Community grant (No. ERB-CIPA-CT-92-0211) during his stay in Grenoble. The authors are grateful to Dr. M.L. Sadowski for a critical reading of the manuscript. We would also like to thank Prof. M. Grynberg for valuable discussions.

\section{References}

[1] T. Strutz, A.M. Witowski, P. Wyder, Rev. Sci. Instrum. 64, 1853 (1993).

[2] K.J. Standley, R.A. Vaughan, Electron Spin Relaxation Phenomena in Solids, Adam Hilger, London 1969.

[3] T. Strutz, A.M. Witowski, P. Wyder, Phys. Rev. Lett. 68, 3912 (1992).

[4] Shen Xue-chu (S.C. Shen), Ye Hong-juan, Kang Li-xue, Tao Feng-xiang, Chin. Phys. Lett. 2, 209 (1985).

[5] A.M. Witowski, S. Oelting, Acta Phys. Pol. A 75, 257 (1989).

[6] A.M. Witowski, W. Bardyszewski, to be published.

[7] Xiaomei Wang, M. Dahl, D. Heiman, P.A. Wolff, P. Becla, Phys. Rev. B 46, 11216 (1992). 